\title{
A Nationwide Assessment of the "July Effect" and Predictors of Post-Endoscopic Retrograde Cholangiopancreatography Sepsis at Urban Teaching Hospitals in the United States
}

\author{
Rupak Desai', Upenkumar Patel ${ }^{2}$, Shreyans Doshi ${ }^{3}$, Dipen Zalavadia ${ }^{4}$, Wardah Siddiq ${ }^{5}$, Hitanshu Dave ${ }^{6}$, Mohammad Bilal ${ }^{7}$, \\ Vikas Khullar ${ }^{8}$, Hemant Goyal ${ }^{9}$, Madhav Desai ${ }^{10}$ and Nihar Shah ${ }^{11}$ \\ ${ }^{1}$ Atlanta VA Medical Center, Decatur, GA, ${ }^{2}$ Department of Internal Medicine, Nassau University Medical Center, East Meadow, NY, \\ ${ }^{3}$ Department of Internal Medicine, College of Medicine/Hospital Corporation of America Graduate Medicine Education Consortium, University \\ of Central Florida, Gainesville, FL, ${ }^{4}$ Department of Internal Medicine, The Wright Center for Graduate Medical Education, Scranton, PA, ${ }^{5}$ Beth \\ Israel Deaconess Medical Center, Harvard Medical School, Boston, MA, ${ }^{6}$ Department of Internal Medicine, Jersey Shore University Medical \\ Center, Neptune, NJ, 'Division of Gastroenterology \& Hepatology, Department of Internal Medicine, University of Texas Medical Branch, \\ Galveston, TX, ${ }^{8}$ Division of Gastroenterology, Department of Internal Medicine, University of Florida, Gainesville, FL, ${ }^{9}$ Department of Internal \\ Medicine, Mercer University School of Medicine, Macon, GA, ${ }^{10}$ Division of Gastroenterology \& Hepatology, Department of Internal Medicine, \\ University of Kansas Medical Center, Kansas City, MO, ${ }^{11}$ Division of Gastroenterology, Department of Internal Medicine, Joan C. Edwards \\ School of Medicine, Marshall University, Huntington, WV, USA
}

Background/Aims: To analyze the incidence of post-endoscopic retrograde cholangiopancreatography (ERCP) sepsis in the early (July to September) and later (October to June) academic months to assess the "July effect".

Methods: The National Inpatient Sample (2010-2014) was used to identify ERCP-related adult hospitalizations at urban teaching hospitals by applying relevant procedure codes from the International Classification of Diseases, 9th revision, Clinical Modification. Post-ERCP outcomes were compared between the early and later academic months. A multivariate analysis was performed to evaluate the odds of post-ERCP sepsis and its predictors.

Results: Of 481,193 ERCP procedures carried out at urban teaching hospitals, 124,934 were performed during the early academic months. The demographics were comparable for ERCP procedures performed during the early and later academic months. A higher incidence $(9.4 \%$ vs. $8.8 \%, p<0.001)$ and odds (odds ratio [OR], 1.07) of post-ERCP sepsis were observed in ERCP performed during the early academic months. The in-hospital mortality rate ( $7 \%$ vs. $7.5 \%, p=0.072)$, length of stay, and total hospital charges in patients with post-ERCP sepsis were also equivalent between the 2 time points. Pre-ERCP cholangitis (OR, 3.20) and post-ERCP complications such as cholangitis $(\mathrm{OR}, 6.27)$, perforation $(\mathrm{OR}, 3.93)$, and hemorrhage $(\mathrm{OR}, 1.42)$ were significant predictors of higher post-ERCP sepsis in procedures performed during the early academic months.

Conclusions: The July effect was present in the incidence of post-ERCP sepsis, and academic programs should take into consideration the predictors of post-ERCP sepsis to lower health-care burden. Clin Endosc 2019;52:486-496

Key Words: Pancreatitis; Endoscopic retrograde cholangiopancreatography; Sepsis; Length of stay; Mortality

Received: October 22, 2018 Revised: February 5, 2019

Accepted: February 27, 2019

Correspondence: Hemant Goyal

Department of Internal Medicine, Mercer University School of Medicine, 707 Pine St. Macon, GA 31201, USA

Tel: +1-478-301-5862, Fax: +1-478-301-5841, E-mail: doc.hemant@yahoo.com ORCID: https://orcid.org/0000-0002-9433-9042

(c) This is an Open Access article distributed under the terms of the Creative Commons Attribution Non-Commercial License (http://creativecommons.org/ licenses/by-nc/3.0) which permits unrestricted non-commercial use, distribution, and reproduction in any medium, provided the original work is properly cited.

\section{INTRODUCTION}

The "July effect" is a hypothesis in academic medicine referring to the potential decline in the quality of care and patient outcomes associated with the enrollment and relative inexperience of new fellows. ${ }^{1,2}$ Each July, academic hospital centers implement a substitution in medical staff, with a large number of centers accepting new interns and fellows to take over patient-care responsibilities. This practice has given rise 
to the belief that a higher number of medical blunders occur in earlier academic months, which may antagonistically affect patient care and in-hospital outcomes. Endoscopic retrograde cholangiopancreatography (ERCP) is a diagnostic and therapeutic maneuver that is typically used to manage biliary and pancreatic diseases. ERCP is a technically demanding procedure involving the biliary and pancreatic ducts. It requires prolonged multidisciplinary care, which likely involves care by interns and fellows-in-training before, during, and after ERCP. There remains a possibility of greater post-ERCP complications during the early phase of fellowship training, considering the undeveloped skill set of new fellows. The most common post-ERCP complications, such as pancreatitis, hemorrhage, perforation, and infections, were noted even with experienced and trained operators. ${ }^{3}$ Freeman et al. mentioned that nearly $10 \%$ of patients who underwent ERCP procedures developed procedure-related complications such as pancreatitis (5.4\%), hemorrhage (2\%), and cholangitis (1.0\%). ${ }^{4}$ Cholangitis predominates among the most dreaded post-ERCP complications that can lead to septicemia. ${ }^{5}$ Post-ERCP mortality due to infection has been noted in up to $8 \%$ of the cases. ${ }^{6}$ Preliminary data have shown that the incidence of post-ERCP sepsis ( $8.8 \%$ vs. $6.3 \%)$ and related mortality ( $7.5 \%$ vs. $6.6 \%)$ is higher in academic hospitals than in non-academic hospitals. ${ }^{7}$ Considering the paucity of data, we aimed to evaluate the existence of the July effect in post-ERCP complications (especially sepsis) at urban teaching hospitals, by using the National Inpatient Sample (NIS) in the United States.

\section{MATERIALS AND METHODS}

\section{Data source}

The study cohort was recruited from the 2010-2014 NIS, which was created and is maintained by the Agency for Healthcare Research and Quality for Healthcare Cost and Utilization Project. ${ }^{8}$ The NIS is the leading all-payer inpatient dataset in the United States, which is publicly obtainable. It is composed by a stratified sample taken from $20 \%$ of the non-federal US community hospitals. When weighted, 95\% of the US population is represented by this dataset. It includes more than 7 million unweighted hospitalizations per year that can be transformed into weighted hospitalization by discharge weight, which is already provided in the database. The weighted dataset comprises more than 35 million hospitalizations per year, which denotes the nationwide estimations. The NIS contains data on the patients' demographics; diagnoses (up to 25 primary and secondary diagnoses); procedures (up to 15 primary and secondary procedures); and hospital features such as ownership, bed size, teaching status, urban/rural setting, and topographical region. Health-care resource utilization data, such as length of stay (LOS), total hospitalization charges, and disposition of patients, are also incorporated into this dataset. The dataset uses the International Classification of Diseases, 9th revision, Clinical Modification (ICD-9 CM) coding system to report up to 25 discharge diagnoses and 15 procedures per discharge. Because the NIS dataset does not include specific identifying information of patients, institutional review board approval for this study was not required.

\section{Study population}

We used the ICD-9 CM procedure codes (51.10, 51.11, 52.13, $52.14,52.21,51.64,52.92,52.93,52.94,52.97,52.98,51.14,51.88$, $51.87,51.86,51.85$, and 51.84 ) to identify adult inpatients who underwent ERCP. Patients were omitted from the study cohort if data about the ERCP procedure were missing or they underwent ERCP before or on the day of admission, and/or if they were admitted to non-teaching urban or rural hospitals. This was done to capture post-ERCP complications exclusive to the current admission and not referring to other etiologies. Post-ERCP complications were recognized by applying the ICD-9 CM codes to the secondary diagnoses of patients who underwent an ERCP procedure, as mentioned above. The same methodology has been used in our prior studies that used the same database to identify procedural complications from secondary discharge diagnoses. ${ }^{2.9-11}$ Post-ERCP sepsis was identified using any of the following codes in the secondary discharge diagnosis field: 0031, 0202, 0223, 0362, 0380, 0381, 03810, 03811, 03812, 03819, 0382, 0383, 03840, 03841, 03842, 03843, 03844, 03849, 0388, 0389, 0545, 449, 77181, 7907, 99591, 99592, 998.51, and 998.59. The ICD-9 CM codes for other ERCP-related complications were reported and approved in the previously published studies. ${ }^{12,13}$ We compared the ERCP procedures performed in the early academic months (July, August, and September) to those performed during the rest of the academic months (October to June) at urban teaching hospitals.

\section{Study variables}

We included the demographics of the study population, such as age, sex, race, type of admission, admission day, payer type, median household income percentile, hospital characteristics (including control/ownership, bed size, and region), and hospitalization outcomes (including in-hospital mortality, LOS, total hospital charges, and disposition of patients). We also incorporated in our analysis the post-ERCP complications such as cholangitis, pancreatitis, perforation, hemorrhage, and sepsis, as well as relevant comorbidities by using the secondary discharge diagnoses. Pre-ERCP cholangitis was identified using the primary discharge diagnoses, whereas post-ERCP 
cholangitis was identified using the secondary discharge diagnoses. This approach has been used in previous NIS studies. ${ }^{12}$

\section{Outcomes}

The primary outcomes were the incidence, in-hospital mortality, LOS, total hospital charges, and disposition of patients with post-ERCP sepsis during the early vs. the later academic months. The secondary outcomes were the predictors of post-ERCP sepsis at urban teaching hospitals and the predictors of post-ERCP sepsis in the procedures performed during the early academic months (July to September) at the urban teaching hospitals.

\section{Statistical analysis}

We used Pearson's chi-square test and Student's $t$-test to evaluate the categorical and continuous variables, respectively, and values were represented as percentages and mean \pm standard deviation, respectively. A 2 -tailed $p$-value of $<0.05$ was used as the cutoff for statistical significance. By using a complex sample module, we used a 2-way hierarchical multivariate analysis to assess the odds of post-ERCP sepsis and the predictors of post-ERCP sepsis in the procedures performed during the early academic months. Multivariate analysis was adjusted for potential confounders including age, sex, race, median household income national quartile for the patient's zip code, and payer status. We described the results of the multivariate analysis in terms of adjusted odds ratio (OR), 95\% confidence interval (CI), and a 2 -tailed $p$-values. SPSS version 22 (IBM Co., Armonk, NY, USA) was used for statistical analyses. We excluded the missing data of all variables in the analysis. We used weighted data to generate nationwide estimates.

\section{RESULTS}

\section{Baseline demographics and hospital characteristics}

We identified 481,193 ERCP procedures carried out at urban teaching hospitals from January 2010 through December 2014. Of these, 124,934 and 356,259 ERCP procedures were performed during the early (July to September) and the later (October to June) academic months, respectively. The demographics and hospital variables were nearly analogous in patients who underwent ERCP during the early vs. the later academic year; the mean age was 59 years, nearly $88 \%$ of the patients were admitted non-electively, and most of the patients were white (>65\%) and women (58\%) in both clusters (Table 1). A total of 42,986 patients who underwent ERCP developed sepsis as a complication. In these patients, 11,773 and 31,212 procedures were carried out during the early (July to
September) and the later (October to June) academic months, respectively. The demographics and hospital characteristics of the patients with post-ERCP sepsis were virtually comparable between those who underwent ERCP during the early academic months (July to September) and those who underwent the procedure in the later academic months (October to June) (Table 2). ${ }^{14}$

\section{All-cause in-hospital mortality and post-ERCP complications}

The rate of all-cause mortality was comparable in patients who underwent ERCP during the early vs. the later academic months ( $1.5 \%$ vs. $1.6 \%, p=0.054)$. The incidence of post-ERCP complications such as pancreatitis $(1.2 \%$ vs. $1.1 \%, p=0.004)$ and sepsis $(9.4 \%$ vs. $8.8 \%, p<0.001)$ was higher during the early than during the later academic months. However, the incidence of post-ERCP hemorrhage ( $1.1 \%$ vs. $1.3 \%, p<0.001)$ was lower during the early than during the later academic months, whereas the incidence of perforation after ERCP was comparable between the early and the later academic months $(0.2 \%$ vs. $0.2 \%, p=0.044$ ) (Table 1). Similarly, the in-hospital mortality rate of post-ERCP sepsis was also comparable between the early $(7 \%)$ and the later $(7.5 \%)$ academic months $(p=0.072)$ (Table 2).

\section{LOS and total hospitalization charges}

There was no difference in LOS (mean 6.8 days vs. 6.8 days, $p=0.04$ ) between the early and the later academic months; however, the total hospital charges after the ERCP procedure at urban teaching hospitals were marginally higher (\$66,688 vs. $\$ 65,105, p<0.001$ ) during the early academic months (Table 1). Correspondingly, there were no significant distinctions in LOS (12.6 \pm 15.1 days vs. $12.7 \pm 15.1$ days, $p=0.359)$ and total hospital charges $(\$ 133,016 \pm \$ 197,740$ vs. $\$ 130,383 \pm \$ 200,670$, $p=0.231$ ) in post-ERCP sepsis cases between the early and the later academic months (Table 2).

\section{Patient disposition}

Patients who developed sepsis after the ERCP procedures performed during the early academic months were more often discharged against medical advice ( $0.3 \%$ vs. $0.2 \%, p=0.001)$ and were more often transferred (to a skilled nursing facility, intermediate care facility, and other facility) ( $24.4 \%$ vs. $23.1 \%$, $p=0.001$ ) than those who underwent ERCP during the rest of the year. Patients with post-ERCP sepsis had fewer routine discharges ( $45.2 \%$ vs. $45.7 \%, p=0.001)$ during the early academic months than during the later academic months (Table 2). 
Table 1. Baseline Characteristics of ERCP Study Population at Urban-Teaching Hospitals between Early vs. Later Academic Year $(n=481,193)$

\begin{tabular}{|c|c|c|c|c|c|}
\hline \multirow[t]{2}{*}{ Variables } & \multicolumn{2}{|c|}{$\begin{array}{c}\text { Early academic year } \\
\text { (July to September) }(n=124,934)\end{array}$} & \multicolumn{2}{|c|}{$\begin{array}{l}\text { Later academic year } \\
\text { (October to June) } \\
\quad(n=356,259)\end{array}$} & \multirow[t]{2}{*}{$p$-value ${ }^{\text {a) }}$} \\
\hline & $n$ & $\%$ & $n$ & $\%$ & \\
\hline Age in years (mean) & \multicolumn{2}{|c|}{59.3} & \multicolumn{2}{|c|}{59} & 0.028 \\
\hline Admission type & & & & & 0.016 \\
\hline Non-elective & 109,588 & $87.8 \%$ & 311,534 & $87.5 \%$ & \\
\hline Elective & 15,230 & $12.2 \%$ & 44,357 & $12.5 \%$ & \\
\hline Indicator of sex & & & & & 0.505 \\
\hline Male & 52,213 & $41.8 \%$ & 148,491 & $41.7 \%$ & \\
\hline Female & 72,680 & $58.2 \%$ & 207,619 & $58.3 \%$ & \\
\hline Race & & & & & 0.012 \\
\hline White & 76,224 & $65.9 \%$ & 216,228 & $65.6 \%$ & \\
\hline African American & 13,008 & $11.2 \%$ & 38,133 & $11.6 \%$ & \\
\hline Hispanic & 17,406 & $15.1 \%$ & 49,065 & $14.9 \%$ & \\
\hline Asian & 4,053 & $3.5 \%$ & 11,911 & $3.6 \%$ & \\
\hline Native American & 739 & $0.6 \%$ & 2,049 & $0.6 \%$ & \\
\hline Others & 4,217 & $3.6 \%$ & 12,235 & $3.7 \%$ & \\
\hline Length of stay in days (mean) & \multicolumn{2}{|c|}{6.8} & \multicolumn{2}{|c|}{6.8} & 0.04 \\
\hline Total hospital charges (mean) & \multicolumn{2}{|c|}{$\$ 66,688$} & \multicolumn{2}{|c|}{$\$ 65,105$} & $<0.001$ \\
\hline \multicolumn{6}{|l|}{ ERCP outcomes } \\
\hline In-hospital mortality & 1,877 & $1.5 \%$ & 5,631 & $1.6 \%$ & 0.054 \\
\hline Post-ERCP pancreatitis & 1,484 & $1.2 \%$ & 3,878 & $1.1 \%$ & 0.004 \\
\hline Post-ERCP perforation & 259 & $0.2 \%$ & 637 & $0.2 \%$ & 0.044 \\
\hline Post-ERCP hemorrhage & 1,407 & $1.1 \%$ & 4,510 & $1.3 \%$ & $<0.001$ \\
\hline Post-ERCP sepsis & 11,773 & $9.4 \%$ & 31,212 & $8.8 \%$ & $<0.001$ \\
\hline
\end{tabular}

ERCP, endoscopic retrograde cholangiopancreatography.

${ }^{\text {a) }}$ Significant $p$-values $<0.05$.

\section{Odds and predictors of post-ERCP sepsis at urban teaching hospitals}

After controlling for plausible confounding factors, ERCP procedures performed during the early academic months (July to September) (OR, 1.07; CI, 1.05-1.10; $p<0.001)$ were found to be a significant predictor of a higher incidence of post-ERCP sepsis. Advanced age ( $\geq 85$ years) (OR, 3.65; CI, 3.48-3.84; $p<0.001$ ), female sex (OR, 1.39; CI, 1.36-1.42; $p<0.001$ ), elective admission (OR, 1.55 ; CI, 1.49-1.61; $p<0.001$ ), Asian/Pacific Islander race (OR, 1.33; CI, 1.26-1.40; $p<0.001$ ), and Native American race (OR, 1.45; CI, 1.27-1.65; $p<0.001$ ) were significant factors associated with a higher incidence of post-ERCP sepsis. Prolonged LOS (>12 days) (OR, 7.23; CI, $6.97-7.50 ; p<0.001)$ and ERCP performed at hospitals in the western region (OR, 1.30; CI, 1.25-1.34; $p<0.001)$ were also significant predictors of a higher incidence of post-ERCP sepsis. Comorbidities such as coagulopathy (OR, 2.33; CI, 2.26-2.40; $p<0.001$ ), uncomplicated diabetes (OR, 1.14; CI, 1.11-1.17; $p<0.001$ ), diabetes with chronic complications (OR, 1.15; CI, 1.09-1.21; $p<0.001$ ), liver disorder (OR, 1.07; CI, 1.03-1.12; $p=0.001$ ), and obesity (OR, 1.12; CI, 1.08-1.16; $p<0.001$ ) significantly increased the odds of post-ERCP sepsis (Table 3).

Predictors of post-ERCP sepsis in procedures performed during the early (July to September) academic months

Advanced age (OR, 1.02; CI, 1.02-1.02; $p<0.001)$, male sex (OR, 1.27; CI, 1.21-1.33; $p<0.001$ ), Hispanic race (OR, 1.09; CI, 1.02-1.17; $p=0.014)$, Asian/Pacific Islander race (OR, 1.32; CI, $1.19-1.46$; $p<0.001$ ), elective admission (OR, 1.25; CI, 1.15-1.35; $p<0.001$ ), and western region location of the hospital (OR, 1.15; CI, 1.07-1.23; $p<0.001$ ) were significant predictors of a higher incidence of post-ERCP sepsis in procedures performed during the early academic months. 
Table 2. Baseline Demographics and Hospital Characteristics of Post-Endoscopic Retrograde Cholangiopancreatography Sepsis Population during Early vs. Later Academic Year $(n=42,986)$

\begin{tabular}{|c|c|c|c|c|c|}
\hline \multirow{2}{*}{$\begin{array}{l}\text { Variables } \\
\text { Age in years mean }( \pm \mathrm{SD})\end{array}$} & \multicolumn{2}{|c|}{ July to September $(n=11,773)$} & \multicolumn{2}{|c|}{ Rest of the year $(n=31,212)$} & $p$-value \\
\hline & \multicolumn{2}{|c|}{$68( \pm 16)$} & \multicolumn{2}{|c|}{$67( \pm 16)$} & 0.01 \\
\hline Sex & & & & & 0.082 \\
\hline Male & 6,242 & $53.0 \%$ & 16,835 & $54.0 \%$ & \\
\hline Female & 5,532 & $47.0 \%$ & 14,368 & $46.0 \%$ & \\
\hline Admission type & & & & & 0.609 \\
\hline Non-elective & 10,852 & $92.3 \%$ & 28,710 & $92.1 \%$ & \\
\hline Elective & 906 & $7.7 \%$ & 2,447 & $7.9 \%$ & \\
\hline Admission day & & & & & 0.062 \\
\hline Weekday (Mon-Fri) & 9,006 & $76.5 \%$ & 23,605 & $75.6 \%$ & \\
\hline Weekend (Sat-Sun) & 2,768 & $23.5 \%$ & 7,607 & $24.4 \%$ & \\
\hline Race & & & & & $<0.001$ \\
\hline White & 7,074 & $65.9 \%$ & 19,063 & $66.4 \%$ & \\
\hline African American & 1,133 & $10.6 \%$ & 3,354 & $11.7 \%$ & \\
\hline Hispanic & 1,396 & $13.0 \%$ & 3,308 & $11.5 \%$ & \\
\hline Asian and Pacific Islander & 641 & $6.0 \%$ & 1,567 & $5.5 \%$ & \\
\hline Native American & 69 & $0.6 \%$ & 255 & $0.9 \%$ & \\
\hline Others & 421 & $3.9 \%$ & 1,150 & $4.0 \%$ & \\
\hline $\begin{array}{l}\text { Median household income percentile for patient's } \\
\text { zip code }{ }^{\text {a) }}\end{array}$ & & & & & $<0.001$ \\
\hline $0-25$ th & 2,826 & $24.5 \%$ & 8,076 & $26.5 \%$ & \\
\hline $26-50$ th & 2,784 & $24.2 \%$ & 7,495 & $24.6 \%$ & \\
\hline $51-75$ th & 2,846 & $24.7 \%$ & 7,411 & $24.3 \%$ & \\
\hline $76-100$ th & 3,057 & $26.6 \%$ & 7,507 & $24.6 \%$ & \\
\hline Primary expected payer & & & & & $<0.001$ \\
\hline Medicare & 7,368 & $62.7 \%$ & 19,015 & $61.0 \%$ & \\
\hline Medicaid & 1,074 & $9.1 \%$ & 3,260 & $10.5 \%$ & \\
\hline Private including HMO & 2,577 & $21.9 \%$ & 7,038 & $22.6 \%$ & \\
\hline Self - Pay/no charge/others & 730 & $6.2 \%$ & 1,864 & $6.0 \%$ & \\
\hline Bed size of hospital & & & & & 0.027 \\
\hline Small & 1,438 & $12.2 \%$ & 3,981 & $12.8 \%$ & \\
\hline Medium & 2,838 & $24.1 \%$ & 7,793 & $25.0 \%$ & \\
\hline Large & 7,497 & $63.7 \%$ & 19,438 & $62.3 \%$ & \\
\hline Region of hospital & & & & & $<0.001$ \\
\hline Northeast & 3,059 & $26.0 \%$ & 7,846 & $25.1 \%$ & \\
\hline Midwest & 3,044 & $25.9 \%$ & 7,623 & $24.4 \%$ & \\
\hline South & 3,391 & $28.8 \%$ & 9,715 & $31.1 \%$ & \\
\hline West & 2,279 & $19.4 \%$ & 6,029 & $19.3 \%$ & \\
\hline \multicolumn{6}{|l|}{ Outcomes } \\
\hline Disposition & & & & & 0.001 \\
\hline Routine & 5,318 & $45.2 \%$ & 14,264 & $45.7 \%$ & \\
\hline Transfer to short-term hospital & 255 & $2.2 \%$ & 813 & $2.6 \%$ & \\
\hline Other transfers (SNF, ICF, other) & 2,876 & $24.4 \%$ & 7,207 & $23.1 \%$ & \\
\hline
\end{tabular}


Table 2. Continued

\begin{tabular}{|c|c|c|c|c|c|}
\hline \multirow{2}{*}{$\begin{array}{l}\text { Variables } \\
\text { Home health care }\end{array}$} & \multicolumn{2}{|c|}{ July to September $(n=11,773)$} & \multicolumn{2}{|c|}{ Rest of the year $(n=31,212)$} & \multirow[t]{2}{*}{$p$-value } \\
\hline & 2,452 & $20.8 \%$ & 6,482 & $20.8 \%$ & \\
\hline Against Medical Advice & 40 & $0.3 \%$ & 65 & $0.2 \%$ & \\
\hline Length of stay (days) mean $( \pm S D)$ & \multicolumn{2}{|c|}{$12.6( \pm 15.1)$} & \multicolumn{2}{|c|}{$12.7( \pm 15.1)$} & 0.359 \\
\hline Total hospital charges mean $( \pm S D)$ & \multicolumn{2}{|c|}{$\$ 133,016( \pm 197,740)$} & \multicolumn{2}{|c|}{$\$ 130,383( \pm 200,670)$} & 0.231 \\
\hline In-hospital mortality & 823 & $7.0 \%$ & 2,340 & $7.5 \%$ & 0.072 \\
\hline
\end{tabular}

The bed size cutoff points distributed into small, medium, and large. It has been done so that nearly one-third of the hospitals in a given region, location, and teaching status combination would fall within each bed size category.

HMO, health maintenance organization; ICF, intermediate care facility; SD, standard deviation; SNF, skilled nursing facility.

${ }^{\text {a) }}$ Represents a quartile classification of the estimated median household income of residents in the patient's ZIP Code, derived from ZIP Code-demographic data obtained from Claritas. ${ }^{14}$

Comorbidities such as coagulopathy (OR, 1.96; CI, 1.84-2.09; $p<0.001$ ), uncomplicated diabetes (OR, 1.10; CI, 1.04-1.16; $p=0.001$ ), diabetes with chronic complications (OR, 1.39; CI, 1.25-1.54; $p<0.001$ ), drug abuse (OR, 1.28; CI, 1.10-1.48; $p=0.001)$, fluid and electrolyte disorders (OR, 1.81; CI, 1.731.90 ; $p<0.001$ ), obesity (OR, 1.07; CI, $1.00-1.15 ; p=0.048)$, renal failure (OR, 1.13; CI, 1.06-1.21; $p<0.001$ ), history of thromboembolism (OR, 1.37; CI, 1.27-1.48; $p<0.001$ ), and pre-ERCP cholangitis (OR, 3.20; CI, 2.91-3.51; $p<0.001)$ were crucial factors for a higher incidence of post-ERCP sepsis in procedures performed during the early academic months. Post-ERCP complications such as cholangitis (OR, 6.27; CI, 5.97-6.58; $p<0.001$ ), perforation (OR, 3.93; CI, 2.90-5.32; $p<0.001$ ), and hemorrhage (OR, 1.42; CI, 1.20-1.67; $p<0.001$ ) also significantly increased the odds of post-ERCP sepsis in procedures performed during the early academic months (Table 4 ).

\section{DISCUSSION}

Although a few studies have determined the outcomes of ERCP, this is the first nationwide study to investigate the July effect in the incidence of post-ERCP sepsis and related hospitalization outcomes. The essential findings of the study were as follows: The incidence of post-ERCP sepsis during the early academic months was $9.4 \%$. The all-cause in-hospital mortality and post-ERCP sepsis-related mortality rates were comparable between the early and the later academic months. There was no difference in the LOS and total hospital charges due to post-ERCP sepsis between the 2 time points. Patients with post-ERCP sepsis were more often transferred to other facilities and had a fewer routine discharges during the early academic months than during the rest of the academic months. ERCP performed during the early academic months was found to be a significant predictor of a higher incidence of post-ERCP sepsis. Pre- and post-ERCP cholangitis, as well as other post-ERCP complications such as perforation and hemorrhage, were major predictors of a higher post-ERCP sepsis incidence in the procedures performed during the early academic months. This large nationwide analysis revealed a higher occurrence of sepsis after in-hospital ERCP at urban academic organizations during the 5 academic years from 2010 through 2014.

Whether post-ERCP sepsis is linked to a faulty procedural technique or overall periprocedural care is still debated. Nonetheless, our result of higher odds of post-ERCP sepsis suggests that close supervision and stringent mentorship and training of fellows and residents are warranted at academic centers in view of the reports of hourly increasing mortality of untreated sepsis. ${ }^{15}$ Contrary to our findings, a study conducted in Romania demonstrated an increasing technical success with continued experience, reflecting the importance of a learning curve; however, the lack of a difference in procedural complications among different levels of operator experience indicates no further risk in procedures performed by supervised fellow trainees. ${ }^{16,17}$ Cannulation time is one of the important markers of technical success in ERCP. One study showed prolonged cannulation time with trainee involvement, without serious adverse outcomes; thus, it can be speculated that a higher procedural time and additional unskilled maneuverers (e.g., sphincterotomy, stone removal, tissue sampling, or stent deployment) could have led to the higher post-ERCP sepsis incidence in our study. ${ }^{18}$

The periprocedural bacteremia rate varies between $15 \%$ and $27 \%$ for ERCP, and the rates are not different for diagnostic or therapeutic ERCP procedures. ${ }^{19}$ In addition to cholangitis, use of combined percutaneous and endoscopic techniques, stent deployment for malignant strictures, concomitant jaundice, failed biliary drainage, and an immunocompromised patient state have been considered as likely explanations for post-ER$\mathrm{CP}$ infections caused by Enterobacteriaceae bacteria in most of the cases. ${ }^{19}$ The guideline for antibiotic prophylaxis before 
Table 3. Multivariate Odds of Post-Endoscopic Retrograde Cholangiopancreatography Sepsis at Urban-Teaching Hospitals

\begin{tabular}{|c|c|c|c|c|}
\hline \multirow{2}{*}{ Predictors } & \multirow{2}{*}{ Odds ratio } & \multicolumn{2}{|c|}{$95 \%$ CI } & \multirow{2}{*}{$p$-value } \\
\hline & & Lower & Upper & \\
\hline Age (yr) at admission & & & & $<0.001$ \\
\hline $45-64$ vs. $18-44$ & 2.01 & 1.93 & 2.10 & $<0.001$ \\
\hline $65-84$ vs. $18-44$ & 2.97 & 2.85 & 3.09 & $<0.001$ \\
\hline$\geq 85$ vs. $18-44$ & 3.65 & 3.48 & 3.84 & $<0.001$ \\
\hline Female vs. Male & 1.39 & 1.36 & 1.42 & $<0.001$ \\
\hline Weekend vs. Weekday admission & 0.91 & 0.89 & 0.93 & $<0.001$ \\
\hline Elective vs. Non-elective admission & 1.55 & 1.49 & 1.61 & $<0.001$ \\
\hline Bed size of hospital & & & & $<0.001$ \\
\hline Medium vs. Small & 0.93 & 0.89 & 0.96 & $<0.001$ \\
\hline Large vs. Small & 0.94 & 0.91 & 0.97 & 0.001 \\
\hline Race & & & & $<0.001$ \\
\hline African American vs. White & 0.99 & 0.96 & 1.03 & 0.659 \\
\hline Hispanic vs. White & 0.95 & 0.92 & 0.99 & 0.012 \\
\hline Asian and Pacific Islander vs. White & 1.33 & 1.26 & 1.40 & $<0.001$ \\
\hline Native American vs. White & 1.45 & 1.27 & 1.65 & $<0.001$ \\
\hline Median household income quartile & & & & $<0.001$ \\
\hline $0-25$ th percentile vs. $76-100$ th percentile & 0.89 & 0.86 & 0.91 & $<0.001$ \\
\hline $26-50$ th percentile vs. $76-100$ th percentile & 0.93 & 0.90 & 0.96 & $<0.001$ \\
\hline 51-75th percentile vs. $76-100$ th percentile & 0.90 & 0.87 & 0.93 & $<0.001$ \\
\hline Hospital region & & & & $<0.001$ \\
\hline Midwest vs. Northeast & 1.05 & 1.02 & 1.09 & 0.003 \\
\hline South vs. Northeast & 1.05 & 1.02 & 1.08 & 0.001 \\
\hline West vs. Northeast & 1.30 & 1.25 & 1.34 & $<0.001$ \\
\hline Length of stay (days) & & & & $<0.001$ \\
\hline $4-6$ vs. $\leq 3$ & 1.93 & 1.86 & 2.00 & $<0.001$ \\
\hline $7-9$ vs. $\leq 3$ & 2.95 & 2.84 & 3.07 & $<0.001$ \\
\hline $10-12$ vs. $\leq 3$ & 3.66 & 3.50 & 3.82 & $<0.001$ \\
\hline$>12$ vs. $\leq 3$ & 7.23 & 6.97 & 7.50 & $<0.001$ \\
\hline \multicolumn{5}{|l|}{ Comorbidities } \\
\hline Alcohol abuse & 0.97 & 0.92 & 1.02 & 0.244 \\
\hline Coagulopathy & 2.33 & 2.26 & 2.40 & $<0.001$ \\
\hline Diabetes, uncomplicated & 1.14 & 1.11 & 1.17 & $<0.001$ \\
\hline Diabetes with chronic complications & 1.15 & 1.09 & 1.21 & $<0.001$ \\
\hline Hypertension & 0.99 & 0.96 & 1.01 & 0.262 \\
\hline Liver disorders & 1.07 & 1.03 & 1.12 & 0.001 \\
\hline Obesity & 1.12 & 1.08 & 1.16 & $<0.001$ \\
\hline Smoking & 0.94 & 0.92 & 0.97 & $<0.001$ \\
\hline July-September vs. Other academic months & 1.07 & 1.05 & 1.10 & $<0.001$ \\
\hline
\end{tabular}

Multivariate regression model is adjusted for age, sex, race, admission day, type, hospital bed size, region, median household income, length of stay and relevant comorbidities.

$\mathrm{CI}$, confidence interval. 
Table 4. Predictors of Post-Endoscopic Retrograde Cholangiopancreatography Sepsis in Procedures Performed during July-September Months at Urban Teaching Hospitals

\begin{tabular}{|c|c|c|c|}
\hline \multirow{2}{*}{ Predictors } & \multirow{2}{*}{ Adjusted OR } & $95 \% \mathrm{CI}$ & $p$-value \\
\hline & & \multicolumn{2}{|l|}{ LL-UL } \\
\hline Age (yr) at admission & & & $<0.001$ \\
\hline $45-64$ vs. $18-44$ & 2.12 & $1.95-2.29$ & $<0.001$ \\
\hline $65-84$ vs. $18-44$ & 2.63 & $2.40-2.89$ & $<0.001$ \\
\hline$\geq 85$ vs. $18-44$ & 3.36 & $3.02-3.73$ & $<0.001$ \\
\hline Male vs. Female & 1.27 & $1.21-1.33$ & $<0.001$ \\
\hline \multicolumn{4}{|l|}{ Race } \\
\hline Hispanic vs. White & 1.09 & $1.02-1.17$ & 0.014 \\
\hline Asian/Pacific Islander vs. White & 1.32 & $1.19-1.46$ & $<0.001$ \\
\hline Weekday vs. Weekend admission & 0.99 & $0.94-1.04$ & 0.605 \\
\hline Elective vs. Non-elective admission & 1.25 & $1.15-1.35$ & $<0.001$ \\
\hline \multicolumn{4}{|l|}{ Hospital characteristics } \\
\hline Government non-federal vs. Private invest-own hospital & 0.83 & $0.75-0.92$ & 0.001 \\
\hline private non-profit vs. Private invest-own hospital & 0.81 & $0.74-0.89$ & $<0.001$ \\
\hline Large vs. Small bed size hospital & 1.01 & $0.94-1.08$ & 0.740 \\
\hline West vs. Northeast region hospital & 1.15 & $1.07-1.23$ & $<0.001$ \\
\hline Length of stay (days) & 1.05 & $1.04-1.05$ & $<0.001$ \\
\hline \multicolumn{4}{|l|}{ Comorbidities and complications } \\
\hline Alcohol abuse & 0.99 & $0.90-1.10$ & 0.906 \\
\hline Coagulopathy & 1.96 & $1.84-2.09$ & $<0.001$ \\
\hline Diabetes, uncomplicated & 1.10 & $1.04-1.16$ & 0.001 \\
\hline Diabetes with chronic complications & 1.39 & $1.25-1.54$ & $<0.001$ \\
\hline Drug abuse & 1.28 & $1.10-1.48$ & 0.001 \\
\hline Hypertension & 0.97 & $0.92-1.02$ & 0.207 \\
\hline Liver disease & 1.03 & $0.95-1.12$ & 0.473 \\
\hline Fluid and electrolyte disorders & 1.81 & $1.73-1.90$ & $<0.001$ \\
\hline Obesity & 1.07 & $1.00-1.15$ & 0.048 \\
\hline Smoking & 1.00 & $0.95-1.06$ & 0.855 \\
\hline Renal failure & 1.13 & $1.06-1.21$ & $<0.001$ \\
\hline Previous thromboembolism & 1.37 & $1.27-1.48$ & $<0.001$ \\
\hline Pre-ERCP cholangitis & 3.20 & $2.91-3.51$ & $<0.001$ \\
\hline Post-ERCP cholangitis & 6.27 & $5.97-6.58$ & $<0.001$ \\
\hline Post-ERCP perforation & 3.93 & $2.90-5.32$ & $<0.001$ \\
\hline Post-ERCP hemorrhage & 1.42 & $1.20-1.67$ & $<0.001$ \\
\hline
\end{tabular}

$P<0.05$ indicates clinical significance. Multivariate regression model is adjusted for age, sex, race, admission day, type, hospital bed size, region, median household income, length of stay and relevant comorbidities.

CI, confidence interval; ERCP, endoscopic retrograde cholangiopancreatography; LL, lower limit; OR, odds ratio; UL, upper limit.

diagnostic or therapeutic procedures such as ERCP is not uniform for all cases. Periprocedural bacteremia can lead to sepsis in immunocompetent patients. Sauter et al. claimed that the rate of periprocedural bacteremia ( $2 \%$ vs. $16 \%, p<0.02$ ) can be reduced with antibiotic prophylaxis as compared with no antibiotic prophylaxis pre-ERCP. ${ }^{20}$ However, the incidence of cholangitis did not decline with pre-ERCP antibiotic prophylaxis. ${ }^{20}$ Thosani et al. suggested considering antibiotic 
prophylaxis in patients undergoing ERCP with single-operator choledochoscopy, particularly in older patients with prior stent placement and those who require intraductal stone lithotripsy. ${ }^{21}$ Guidelines for antibiotic prophylaxis before and/ or during the ERCP procedure that are confusing for gastrointestinal medicine fellows could be one of the factors responsible for the higher incidence of post-ERCP sepsis during the early academic months.

The mortality rate in hospitalized patients with sepsis is more than 8 times during their hospital stay. ${ }^{22}$ However, our study found that the all-cause in-hospital mortality and post-ERCP sepsis-related mortality rates were comparable between the early and the rest of the academic months. This suggests that a high proportion of sepsis cases were managed well enough, avoiding higher inpatient mortality and indicating improving critical care in recent times. We found that comorbidities such as diabetes, coagulopathy, liver disorder, and obesity are linked to an increased risk of post-ERCP sepsis, likely owing to their pro-inflammatory nature. ${ }^{23}$ Smoking was not an independent predictor of post-ERCP sepsis as a whole in urban teaching hospital procedures or especially during procedures performed from July to September. Future studies may elucidate this intersection of the smoking paradox in the outcomes of ERCP or the July effect. Advanced age, female sex, Asian/Pacific Islander race, Native American race, and procedures performed in western region hospitals were also associated with higher odds of post-ERCP sepsis. These sex and racial discrepancies in post-ERCP sepsis merit further investigations to confirm our study findings. Prior cholangitis is a well-established predictive factor for the development of sepsis. ${ }^{24}$ Our study also revealed that pre-ERCP cholangitis increased the risk of post-ERCP sepsis by 3 times during the early academic months. Post-ERCP complications such as cholangitis, acute pancreatitis, hemorrhage, and perforation can carry a risk of bacteremia and can progress to severe infectious complications. ${ }^{3}$ As expected, we also observed that pre-ERCP (OR, 3.20) and post-ERCP (OR, 6.27) cholangitis, perforation (OR, 3.93), and hemorrhage (OR, 1.42) increased the risk of septic complications in the ERCP procedures performed during the early academic months at the urban teaching hospitals.

Several studies have assessed and established the existence of the July effect among surgical trainees; ${ }^{25}$ however, the data on the incidence of post-ERCP outcomes, especially post-ER$\mathrm{CP}$ sepsis, among gastrointestinal medicine fellows are trivial. No study using the NIS has shown any difference in the occurrence of post-ERCP pancreatitis during earlier academic months; ${ }^{1}$ however, Cheng et al. revealed that $\mathrm{ERCP}$ procedures that involved trainees had a higher risk for the development of post-ERCP pancreatitis. ${ }^{26}$ Correspondingly, this would be the first study to establish the existence of the July effect in the incidence of post-ERCP sepsis.

Several studies have assessed the LOS and hospitalization charges for various admission diagnoses as a sign of the July effect. ${ }^{27}$ A single-center study over 7 years proved a significant and steady decrease in both total hospital charges and LOS for various types of diagnoses over the academic year. ${ }^{28}$ The difference in the post-ERCP sepsis incidence for the first quarter can have a large impact on the ERCP outcomes in terms of LOS and total hospital charges; however, in patients with post-ERCP sepsis, we have not identified any difference in LOS and total hospital charges between those who underwent the procedure in the early academic months and those who underwent ERCP performed in the later academic months.

Involvement of inexperienced trainees in ERCP procedures and inadequate post-ERCP care can pose a risk of higher complications and, consequently, higher health-care expenditures. Our results demonstrated the existence of the July effect in post-ERCP sepsis. These results suggest that vigilant supervision is needed in ERCP procedures performed by new endoscopists or fellowship trainees, and that it is necessary to follow the recommended minimum procedure $(\sim 200)$ criteria by the American Society for Gastrointestinal Endoscopy to allow new endoscopists to develop full competency in performing the procedures autonomously without major complications. ${ }^{29} \mathrm{~A}$ well-rounded knowledge of the procedural techniques and antibiotic regimens may help in the treatment of complicated post-ERCP sepsis and prevent further inpatient mortality.

One of the biggest strengths of this study is that the NIS is the largest health-care dataset in the United States, which allowed us to evaluate the health-care practice patterns at the national level. Selection and participation biases are common limitations of smaller studies; however, these biases can be minimized given that the sample was taken from a broad range of patient demographics and hospitals from almost every state. Additionally, the results of the study can be generalized to the whole US population. However, our study also has limitations. The operational definitions of post-ERCP complications could have been more accurate with the availability of laboratory data; however, in the absence of laboratory values, we utilized the validated ICD-9 codes used in previous studies. Owing to the retrospective nature of the study, the ability to remove confounders is limited compared with a randomized study. As the NIS largely represents community hospitals, our study might have included data from some non-training programs while excluding fellowship programs from university institutions. Unfortunately, the retrospective nature of the NIS data does not provide sufficient subgroup information to support the sex-based differences in post-ERCP sepsis or 
the lower odds of weekend- and non-elective admission-related sepsis; however, the lower severity of cases or the fewer non-elective procedures being performed on weekends could be factors contributing to the observed findings. As coagulopathy and liver disorders could be inclusive of each other, the results should be interpreted accordingly. We could not gather information on the total procedure time, use of sphincterotomy, degree of supervision by the attending physician, choice of antibiotics, use of prophylactic antibiotics, and other factors. Finally, ICD-9 CM coding errors have been shown to exist in the NIS data. ${ }^{30}$ However, as such errors are arbitrarily dispersed, they should not be a source of bias.

In summary, by using the largest nationwide study cohort, we established the existence of the July effect in the incidence of post-ERCP sepsis and demonstrated that knowledge of the predictors of post-ERCP sepsis may help improve the training curriculum of academic programs. This, in turn, will help in improving the proficiency of fellows or in modifying the pre- and post-ERCP care protocol to reduce the sepsis-related health-care burden at urban teaching hospitals during the early months of the academic year.

\section{Conflicts of Interest}

The authors have no financial conflicts of interest.

\section{Author Contributions}

Conceptualization: Rupak Desai, Upenkumar Patel, Shreyans Doshi

Data curation: RD, UP, SD

Formal analysis: $\mathrm{RD}, \mathrm{UP}, \mathrm{SD}$

Software: RD, UP, SD

Supervision: RD, UP, SD

Validation: RD, UP, SD

Visualization: RD, UP, SD

Writing-original draft: $\mathrm{RD}, \mathrm{UP}, \mathrm{SD}$

Writing-review\&editing: RD, UP, SD, Dipen Zalavadia, Wardah Siddiq, Hitanshu Dave, Mohammad Bilal, Vikas Khullar, Hemant Goyal, Madhav Desai, Nihar Shah

\section{REFERENCES}

1. Schulman AR, Abougergi MS, Thompson CC. Assessment of the July effect in post-endoscopic retrograde cholangiopancreatography pancreatitis: Nationwide Inpatient Sample. World J Gastrointest Endosc 2017;9:296-303.

2. Desai R, Patel U, Goyal H. Does "July effect" exist in colonoscopies performed at teaching hospitals? Transl Gastroenterol Hepatol 2018;3:28.

3. Szary NM, Al-Kawas FH. Complications of endoscopic retrograde cholangiopancreatography: how to avoid and manage them. Gastroenterol Hepatol (N Y) 2013;9:496-504.

4. Freeman ML, Nelson DB, Sherman S, et al. Complications of endoscopic biliary sphincterotomy. N Engl J Med 1996;335:909-918.

5. Classen DC, Jacobson JA, Burke JP, Jacobson JT, Evans RS. Serious Pseudomonas infections associated with endoscopic retrograde cholangiopancreatography. Am J Med 1988;84(3 Pt 2):590-596.
6. Andriulli A, Loperfido S, Napolitano G, et al. Incidence rates of post-ERCP complications: a systematic survey of prospective studies. Am J Gastroenterol 2007;102:1781-1788.

7. Desai R, Doshi S, Patel U, Siddiq W, Patel S, Shah N. Impact of hospital location and teaching status on post-endoscopic retrograde cholangiopancreatography (ERCP) sepsis incidence and outcomes: a 5-year national inpatient outlook. Gastroenterology 2018;154(6 Suppl 1):S1070-S1071.

8. Healthcare Cost and Utilization Project (HCUP). NIS Overview [Internet]. Rockville (MD): HCUP Databases; c2018 [updated 2018 Aug 13; cited 2019 Apr 13]. Available from: https://www.hcup-us.ahrq.gov/ nisoverview.jsp.

9. Desai R, Parekh T, Singh S, et al. Alarming increasing trends in hospitalizations and mortality with Heyde's syndrome: a nationwide inpatient perspective (2007 to 2014). Am J Cardiol 2019;123:1149-1155.

10. Desai R, Patel U, Fong HK, et al. Modern-day nationwide utilization of intravascular ultrasound and its impact on the outcomes of percutaneous coronary intervention with coronary atherectomy in the United States. J Ultrasound Med 2019 Jan 4 [Epub]. https://doi.org/10.1002/ jum.14922.

11. Desai R, Singh S, Fong HK, et al. Racial and sex disparities in resource utilization and outcomes of multi-vessel percutaneous coronary interventions (a 5-year nationwide evaluation in the United States). Cardiovasc Diagn Ther 2019;9:18-29.

12. Inamdar S, Sejpal DV, Ullah M, Trindade AJ. Weekend vs. weekday admissions for cholangitis requiring an ERCP: comparison of outcomes in a national cohort. Am J Gastroenterol 2016;111:405-410.

13. Allareddy V, Rampa S, Rotta AT, Allareddy V. The impact of septicemia occurring during hospitalization for renal transplantation procedures on outcomes in adults in United States. PLoS One 2017;12:e0179466.

14. Healthcare Cost and Utilization Project (HCUP). NIS description of data elements [Internet]. Rockville (MD): HCUP Databases; c2008 [updated 2008 Sep 17; cited 2019 Apr 13]. Available from: https://www. hcup-us.ahrq.gov/db/vars/zipinc_qrtl/nisnote.jsp.

15. Ferrer R, Martin-Loeches I, Phillips G, et al. Empiric antibiotic treatment reduces mortality in severe sepsis and septic shock from the first hour: results from a guideline-based performance improvement program. Crit Care Med 2014;42:1749-1755.

16. Voiosu T, Bengus A, Voiosu A, et al. Trainee caseload correlates with ERCP success rates but not with procedure-related complications: results from a prospective study (the QUASIE cohort). Endosc Int Open 2016;4:E409-E414.

17. Voiosu T, Voiosu A, Benguş A, Rimbaş M, Mateescu B. Trainee involvement increases precut rates and delays access to the common bile duct without an increase in procedure-related adverse events: a brave new world of ERCP training? Rom J Intern Med 2018;56:55-61.

18. Frost JW, Kurup A, Shetty S, Fisher N. Does the presence of a trainee compromise success of biliary cannulation at ERCP? Endosc Int Open 2017;5:E559-E562.

19. Kullman E, Borch K, Lindström E, Anséhn S, Ihse I, Anderberg B. Bacteremia following diagnostic and therapeutic ERCP. Gastrointest Endosc 1992;38:444-449.

20. Sauter G, Grabein B, Huber G, Mannes GA, Ruckdeschel G, Sauerbruch T. Antibiotic prophylaxis of infectious complications with endoscopic retrograde cholangiopancreatography. A randomized controlled study. Endoscopy 1990;22:164-167.

21. Thosani N, Zubarik RS, Kochar R, et al. Prospective evaluation of bacteremia rates and infectious complications among patients undergoing single-operator choledochoscopy during ERCP. Endoscopy 2016;48:424431.

22. Hall MJ, Williams SN, DeFrances CJ, Golosinskiy A. Inpatient care for septicemia or sepsis: a challenge for patients and hospitals. NCHS Data Brief 2011:1-8.

23. Holder AL, Gupta N, Lulaj E, et al. Predictors of early progression to severe sepsis or shock among emergency department patients with 
nonsevere sepsis. Int J Emerg Med 2016;9:10.

24. Motte S, Deviere J, Dumonceau JM, Serruys E, Thys JP, Cremer M. Risk factors for septicemia following endoscopic biliary stenting. Gastroenterology 1991;101:1374-1381.

25. Watkins AA, Bliss LA, Cameron DB, Eskander MF, Tseng JF, Kent TS Deconstructing the "July effect" in operative outcomes: a national study. J Gastrointest Surg 2016;20:1012-1019.

26. Cheng CL, Sherman S, Watkins JL, et al. Risk factors for post-ERCP pancreatitis: a prospective multicenter study. Am J Gastroenterol 2006;101:139-147.

27. Barry WA, Rosenthal GE. Is there a July phenomenon? The effect of
July admission on intensive care mortality and length of stay in teaching hospitals. J Gen Intern Med 2003;18:639-645.

28. Rich EC, Gifford G, Luxenberg M, Dowd B. The relationship of house staff experience to the cost and quality of inpatient care. JAMA 1990;263:953-957.

29. ASGE Standards of Practice Committee, Faulx AL, Lightdale JR, et al. Guidelines for privileging, credentialing, and proctoring to perform GI endoscopy. Gastrointest Endosc 2017;85:273-281.

30. Berthelsen CL. Evaluation of coding data quality of the HCUP National Inpatient Sample. Top Health Inf Manage 2000;21:10-23. 